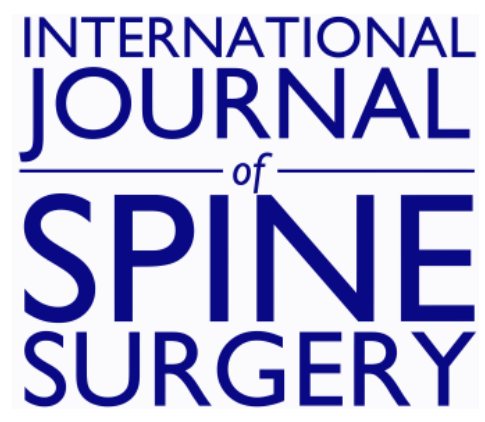

\title{
The 2-Level Experience of Interlaminar Stabilization: 5-Year Follow-Up of a Prospective, Randomized Clinical Experience Compared to Fusion for the Sustainable Management of Spinal Stenosis
}

RACHEL B. SIMON, CHRISTINA DOWE, SAMUEL GRINBERG, FRANK P. CAMMISA, JR and CELESTE ABJORNSON

Int J Spine Surg 2018, 12 (4) 419-427

doi: https://doi.org/10.14444/5050

http://ijssurgery.com/content/12/4/419

This information is current as of April 26, 2023.

Email Alerts Receive free email-alerts when new articles cite this article. Sign up at:

http://ijssurgery.com/alerts

The International Journal of Spine Surgery

2397 Waterbury Circle, Suite 1,

Aurora, IL 60504, Phone: +1-630-375-1432 


\title{
The 2-Level Experience of Interlaminar Stabilization: 5-Year Follow-Up of a Prospective, Randomized Clinical Experience Compared to Fusion for the Sustainable Management of Spinal Stenosis
}

\author{
RACHEL B. SIMON, BA, CHRISTINA DOWE, BS, SAMUEL GRINBERG, BA, FRANK P. CAMMISA JR., \\ MD, CELESTE ABJORNSON, PhD \\ Hospital for Special Surgery, New York, New York
}

\begin{abstract}
Background: To alleviate the symptoms of lumbar spinal stenosis, widely accepted methods of surgical treatment include decompression alone and decompression with fusion. As an alternative to these methods, interlaminar stabilization (ILS) devices with decompression were introduced. There is a large amount of research dedicated to examining the efficacy of ILS devices in single-level procedures, but fewer studies focus on their efficacy in 2-level procedures. The purpose of this study was to compare decompression with instrumented posterolateral fusion to decompression with interlaminar stabilization in patients who require surgical treatment at 2 levels for lumbar spinal stenosis at 5 years postoperation.

Methods: Of the 322 patients enrolled in the Investigational Device Exemption clinical trial, 116 required surgical treatment at 2 levels. The ILS group consisted of 77 patients, and the fusion group consisted of 39 patients. Efficacy was measured using composite clinical success (CCS). Patients achieve CCS if they achieve all 4 of the following outcomes: $\geq 15$-point improvement from baseline Oswestry Disability Index (ODI); no reoperation or epidural injections; no persistent, new, or increasing neurological deficits; and no major device-related complications.

Results: There was a $91 \%$ rate of follow-up within the participant population in the 5-year data. There was a difference trending toward significance between groups for the absence of reoperation or epidural injection, with $68.8 \%$ of ILS patients and only $51.3 \%$ of fusion patients meeting this criteria $(P=.065) ; 13.0 \%$ of ILS patients and $25.7 \%$ of fusion patients required secondary surgery. The percentage of patients achieving overall CCS was much greater in the ILS group than the fusion group, with 55.1\% (38/69) of ILS patients and only 36.4\% (12/33) of fusion patients achieving CCS at month $60(P=.077)$. With regard to the ODI, the visual analog scale back and worse leg pain, the Short Form-12, and the Zurich Claudication Questionnaire, both groups had significantly better results at every followup time point when compared to their respective baseline scores.

Conclusions: The 2-level ILS patient group performed as well as, if not better than, the 2-level fusion group across almost all outcome measures, demonstrating both clinical outcome success and favorably low reoperation rates in patients who received ILS surgery.

Clinical Relevance: This is the first 5-year analysis of the 2-level ILS experience, which supplements previous studies that describe the advantages of ILS by extending such advantages to 2-level cases.
\end{abstract}

New Technology

\section{INTRODUCTION}

Although the symptomatology of lumbar spinal stenosis is widely understood as the result of mechanical pressure on the nerve leading to pain and/or numbness, the care continuum is of significant debate. There are various methods of conservative care to manage the resultant symptoms of spinal stenosis, such as medical pain management, physical therapy, epidural steroid injections, and lifestyle modifications. However, these paths of care do not often provide the patient with sustained improvement of symptoms. Moreover, the 2008 SPORT study ${ }^{1}$ provides evidence that a surgical method is more effective than continuous nonoperative care in patients with symptomatic spinal stenosis persisting for longer than 12 weeks.

Alternative surgical options to decompression alone or decompression with fusion for the treatment of spinal stenosis have been of significant interest during the past decade. Interspinous distraction spacers and interlaminar stabilization (ILS) 
devices differ in mode of action but were developed as a result of this focus. Studies on interspinous spacers, such as X-STOP, have shown no significant difference between conventional decompression and indirect decompression with an interspinous device. $^{2-5}$ In contrast, studies conducted on interlaminar stabilization have provided level I evidence for significantly superior clinical improvements with interlaminar stabilization after decompression compared to posterior fusion with pedicle screw fixation. ${ }^{6-9}$ It has also been shown that ILS can sustain similar results when utilized in multilevel procedures when compared to single-level procedures. $^{9}$ This is of particular importance, as ILS surgery is less invasive than traditional posterior fusion surgical approaches.

Utilizing the Food and Drug Administration (FDA) Investigational Device Exemption (IDE) clinical trial database, the purpose of this study was to perform a cohort analysis of patients who require surgical treatment for lumbar spinal stenosis at 2 levels with 5-year follow-up. The study compared the treatment options of decompression and posterolateral fusion (fusion) to decompression and ILS to determine the rates of long-term success in treating spinal stenosis at 2 levels.

\section{METHODS}

\section{Study Design}

Fully described in the publication by Davis et al, ${ }^{6}$ the study design is a multicenter, prospective, randomized, controlled trial that was conducted at 21 sites in the United States, all under institutional review board approval. Final follow-up was completed in January 2015, with a 5-year follow-up rate of $91 \%$. Patients were blinded until after surgical treatment.

\section{Surgical Technique and coflex Interlaminar Stabilization Device}

The current study focuses on 2 methods of decompressive surgery with stabilization to treat 2 levels of lumbar spinal stenosis. All study subjects, therefore, received a microsurgical decompression, immediately followed by either instrumented fusion (control group) or interlaminar stabilization (investigational group) using coflex (Paradigm Spine, New York, NY). The coflex ILS device achieved FDA premarket approval for up to a Grade I spondylolisthesis in 2012. It is a U-shaped, titanium alloy implant that, after decompression, is fixed between lamina with the apex oriented anteriorly 1-2 mm from the dura and the 2 long arms of the "U" paralleling the long axis of the spinous processes. The goals of implanting the device in the interlaminar space are to unload the facet joints, stabilize the motion segment, maintain the neurological decompression and foraminal height, and preserve some motion. Patients in the control group received a posterolateral fusion with pedicle screws.

\section{Patient Population}

Patients were enrolled using computer-generated randomization codes according to a 2:1 ratio of investigational to control groups. Site study personnel were blinded to assignment of treatment until 5 days before surgery. Study subjects were blinded to treatment assignment until after surgery. Fully described in Davis et al, ${ }^{6}$ briefly, the inclusion and exclusion criteria required patients to be between the ages of 40 and 80 years with moderate to severe lumbar stenosis at 1 or 2 contiguous levels and up to grade I spondylolisthesis, minimum Oswestry Disability Index (ODI) score of $40 \%$ or higher, visual analog scale (VAS) back pain score at least 50 of 100 , and confirmed leg, buttock, or groin pain. The current cohort study investigates the patients treated for 2-level disease. Surgeons ensured that the symptomatology on presentation correlated to the clinical and radiographic findings of spinal stenosis and that patients met all inclusion/exclusion criteria.

\section{Clinical Outcomes Measures}

Standard clinical outcomes assessments were made at baseline as well as postoperatively at 6 weeks, and at 3, 6, 12, 18, 24, 48, and 60-month time points. The following outcomes measures were evaluated: ODI, VAS separately assessing back and leg pain, the Zurich Claudication Questionnaire (ZCQ), and the Short Form-12 (SF-12). To assess neurological outcomes, motor, sensory, and reflex assessments were performed. A neurological outcome was deemed successful if there was maintenance or improvement when comparing postoperative to preoperative assessments. Composite clinical success (CCS), as defined in the FDA IDE protocol, required a patient to meet the following 4 criteria: (1) improvement of at least 15 points in ODI (ODI-15) at 60 months compared with baseline; (2) no reoperations, revisions, removals, or supplemental fixation; (3) no major devicerelated complications, including but not limited to 
permanent, new, or increasing sensory or motor deficit at 60 months; and (4) no lumbar epidural steroid injection at any postoperative time point. If a patient had either a secondary procedure or an injection, the patient was deemed a failure and was excluded from the analysis. Every end point was evaluated at each time point for patients who had not had an epidural injection or secondary procedure. In addition to the minimum 15-point improvement on ODI scores as a measure of favorable clinical outcome for a patient, the percentage of patients improving at least 20 points on the back and leg pain VAS scores was calculated and compared between the 2 groups. The patient accountability chart shows patient flow (Figure 1).

\section{Radiographic Outcomes Measures}

For patients who were assigned decompression with interlaminar stabilization, upright neutral lateral, flexion, and extension radiographs were obtained at each time point. For patients in the decompression with fusion control group, the same radiographic data were obtained, with the exception of flexion and extension radiographs being withheld at the 6-week and 3-month time points. All radiographic images were sent directly from the study sites to an independent core radiography laboratory (Medical Metrics Inc, Houston, Texas) for evaluation.

\section{Statistical Analysis}

Month 60 was the primary efficacy end point for this study. Patients had to have a successful outcome in all 4 end points in order to achieve month 60 CCS. Furthermore, linear improvements were analyzed for each patient-derived questionnaire. Statistical group comparisons included $t$ tests for comparing means, computation of standardized effect sizes (mean difference divided by pooled standard deviation), chi-square, and Fisher exact tests to compare categorical outcomes, graphical analyses, and correlational analyses. The analysis of patient accountability revealed a 60 -month clinical and radiographic follow-up rate of $91 \%$.

\section{RESULTS}

\section{CCS}

At the 5-year follow-up, the percentage of patients who achieved CCS who received decompression and ILS was $55.1 \%$, and the percentage of patients who received decompression and fusion was $36.4 \%$. While approximately a $20 \%$ difference between treatments achieving CCS was not statistically significant, there was a trend favoring ILS patients achieving success over fusion patients $(P=$ .077) (Table 1). Interestingly, if epidural steroid injections (ESI) is removed from the failure definition, the success rates increased from $55.1 \%$ to $69.2 \%$ (diff $=14.1 \%$ ) for ILS patients and from $36.4 \%$ to $53.1 \%$ (diff $=16.7 \%)$ among fusion patients but does not change the overall findings.

\section{$O D I$}

Of those assessed, $86.7 \%$ of ILS patients (39/45) and $92.9 \%$ of fusion patients (13/14) saw an improvement of $\geq 15$ points in the ODI at month 60 compared to baseline $(P=1.0)$. Overall, patients in both groups for 2-level procedures achieved improvement in ODI score from baseline to 60 months. There was no significant difference in 5-year mean ODI scores between ILS and fusion patients $(P=.6)$. However, notably at 6 weeks follow-up, 2-level ILS patient mean ODI scores improved by 35.2 points, while 2-level fusion patient mean ODI scores improved by 25.8 points, rendering a statistically significant difference between groups $(P=.023)$. Within both the ILS and the fusion groups, every follow-up mean ODI score was significantly better than baseline $(P<.001)$ (Figure 2$)$.

\section{Secondary Surgical Procedures and Epidural Steroid Injections}

Overall, there was a large difference trending toward significance between the number of ILS patients who did not require reoperation or epidural steroid injection by month 60 in comparison to fusion patients. The number of patients in the ILS group who did not receive a reoperation or epidural injection was $53 / 77(68.8 \%)$ compared to $20 / 39$ $(51.3 \%)$ patients in the fusion group $(P=.065)$. There was no significant difference between groups for the absence of epidural injection at any lumbar level up to and including the month 60 follow-up visit (80.5\% ILS vs. $69.2 \%$ fusion, $P=.174$ ). Approximately twice as many fusion patients, $25.7 \%$, required reoperations, revisions, removals, or supplemental fixation compared to $13.0 \%$ of ILS patients $(P=.088)$. Ten patients in each group underwent reoperations, revisions, removals, or supplemental fixation for various reasons (Table 2). Focusing on sustainability, the fusion group reported the most reoperation cases $(10.3 \%)$ in the "device 


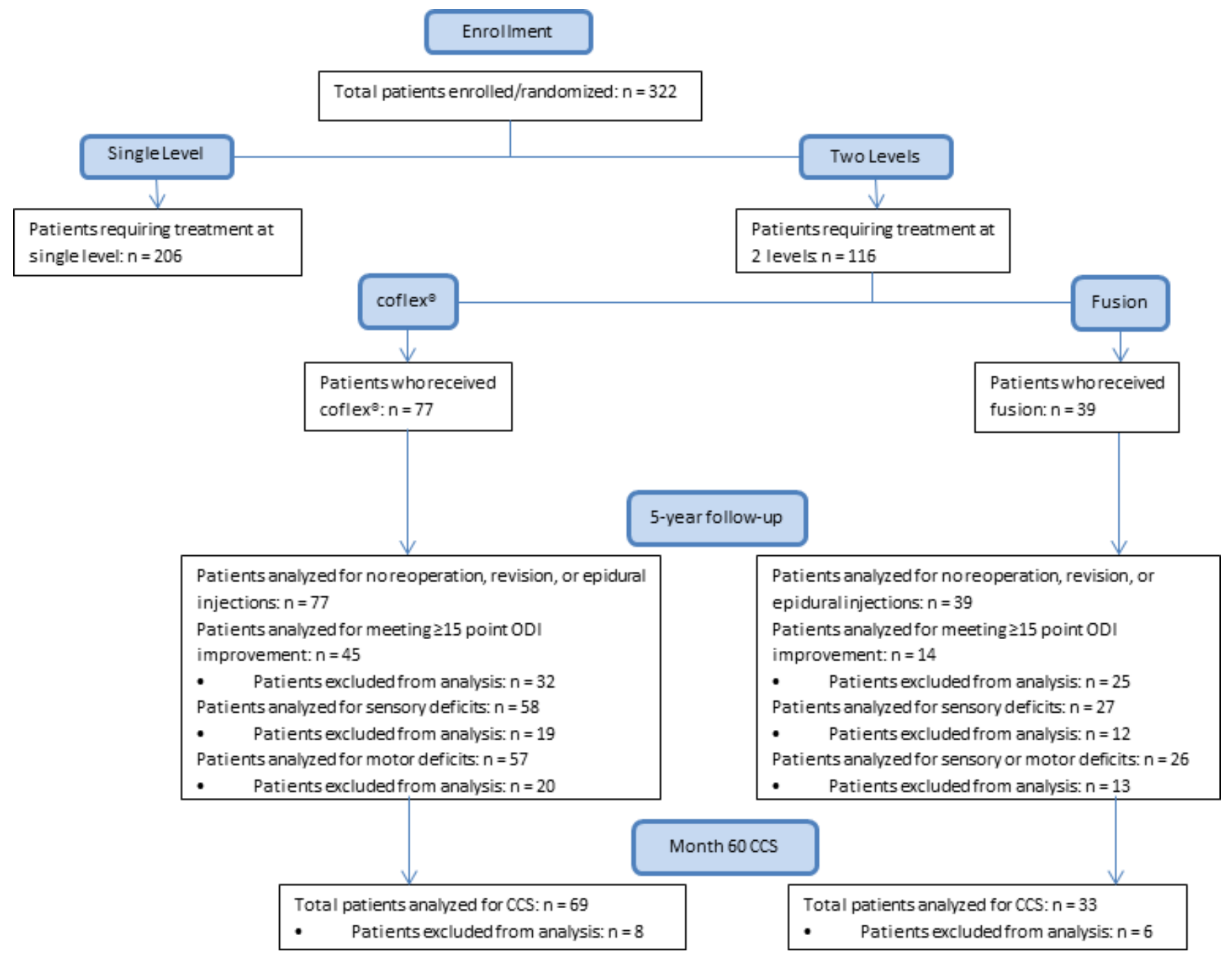

Figure 1. Patient flowchart.

ineffective: late" category, meaning the reoperation was a result of failed fusion or hardware. In comparison, the ILS group reported only 1 case $(1.3 \%)$ in the "device ineffective: late" category.

\section{Neurological Success}

There was no significant difference between groups with 55/57 ILS patients and 25/26 fusion patients having no persistent, new, or increasing sensory or motor deficits at month $60(P>.9)$. When separated, 57/58 ILS patients and 27/27 fusion patients had no persistent, new, or increasing sensory deficits $(P>.4)$, while $56 / 57$ ILS patients and 25/26 fusion patients had no persistent, new, or increasing motor deficits $(P>.5)$.

\section{Major Device-Related Complications}

Two patients in each group experienced major device-related complications. This resulted in
$97.4 \%$ of ILS patients and $94.9 \%$ of fusion patients free of a major device-related complication $(P=$ $.480)$.

\section{Secondary End Points}

\section{VAS}

At 60 months, the mean VAS back pain scores decrease of 59.8 points for the ILS group was similar to the fusion group with 58.9 points. Within both the ILS and the fusion groups, every follow-up mean VAS back pain score was statistically significantly different than baseline based on a withingroup paired $t$ test (ILS $P<.001$, fusion $P<.02$ ). The difference in the 2 groups' 5-year follow-up VAS back pain scores was not statistically significant (20.7 ILS vs. 20.9 fusion, $P>$.9) (Figure 3a).

For VAS worse leg pain (leg that is most symptomatic), while there was no significant differ- 


\section{0}

\section{Mean ODI}

90.0

80.0

70.0

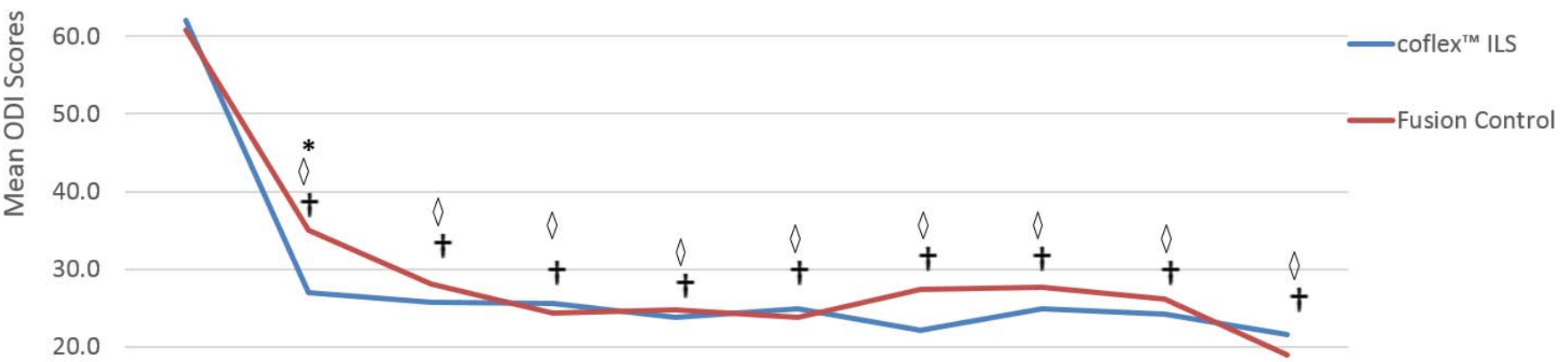

10.0

0.0
Pre-0p
Week
Month 3 Month Follow-Up Time Points

Figure 2. Mean Oswestry Disability Index scores. * Significant difference between interlaminar stabilization (ILS) and fusion groups: 2 sample pooled $t$ test $P$ value $=$ .023. †Significant difference between ILS baseline and follow-up: within-group paired $t$ test. $\diamond$ Significant difference between fusion baseline and follow-up: withingroup paired $t$ test.

Table 1. Overall efficacy. Mean 60 overall efficacy in the extended follow-up physical analysis set descriptive comparisons of the percentages of subjects achieving clinical success defined by the individual components and related variables 2 -level procedures. ${ }^{1}$

\begin{tabular}{|c|c|c|c|c|c|c|c|}
\hline & \multicolumn{6}{|c|}{ Number and Percent Meeting Criteria } & \multirow[b]{3}{*}{$P$ Value } \\
\hline & \multicolumn{3}{|c|}{ Coflex } & \multicolumn{3}{|c|}{ Fusion } & \\
\hline & $\mathbf{N}$ & $\mathbf{n}$ & $(n / N) \%$ & $\mathbf{N}$ & $\mathbf{n}$ & $(n / N) \%$ & \\
\hline Improvement of at least 15 points in ODI at month 60 compared to baseline & 45 & 39 & 86.7 & 14 & 13 & 92.9 & .532 \\
\hline No reoperation or epidural (up to day 1825 ) & 77 & 53 & 68.8 & 39 & 20 & 51.3 & .065 \\
\hline No operations, revisions, removals, or supplemental fixation & 77 & 67 & 87.0 & 39 & 29 & 74.4 & .088 \\
\hline No epidural injection at any lumbar level up to and including the month 60 visit & 77 & 62 & 80.5 & 39 & 27 & 69.2 & .174 \\
\hline No persistent, ${ }^{2}$ new, or increasing sensory or motor deficit at 60 months & 57 & 55 & 96.5 & 26 & 25 & 96.2 & .939 \\
\hline No persistent, ${ }^{2}$ new, or increasing sensory deficit at 60 months & 58 & 57 & 98.3 & 27 & 27 & 100.0 & .493 \\
\hline No persistent, ${ }^{2}$ new, or increasing motor deficit at 60 months & 57 & 55 & 96.5 & 26 & 25 & 96.2 & .939 \\
\hline No major device-related complications & 77 & 75 & 97.4 & 39 & 37 & 94.9 & .480 \\
\hline Composite clinical success (month 60 CCS-FDA) & 69 & 38 & 55.1 & 33 & 12 & 36.4 & .077 \\
\hline \multicolumn{8}{|l|}{ Other related outcomes } \\
\hline Increase of $20 \mathrm{~mm}$ on leg pain VAS & 43 & 43 & 100.0 & 13 & 13 & 100.0 & \\
\hline Maintenance or improvement of the SF-12 PCS score & 40 & 35 & 87.5 & 12 & 10 & 83.3 & .711 \\
\hline ZCQ symptom severity improves at least 0.5 & 45 & 38 & 84.4 & 14 & 12 & 85.7 & .908 \\
\hline ZCQ physical function improves at least 0.5 & 45 & 37 & 82.2 & 14 & 11 & 78.6 & .759 \\
\hline Patient at least somewhat satisfied ${ }^{2}$ & 44 & 41 & 93.2 & 14 & 13 & 92.9 & .967 \\
\hline Patient would definitely or probably recommend same treatment ${ }^{3}$ & 44 & 41 & 93.2 & 14 & 12 & 85.7 & .386 \\
\hline
\end{tabular}

Abbreviations: ODI, Oswestry Disability Index; CCS, composite clinical success; FDA, Food and Drug Administration; VAS, visual analog scale; SF-12, Short Form-12; PCS; Physical Component Summary; ZCQ, Zurich Claudication Questionnaire.

${ }^{1}$ Chi-square test.

${ }^{2}$ Patient satisfaction. Very satisfied + somewhat satisfied versus somewhat dissatisfied + very dissatisfied.

${ }^{3}$ Definitely yes + probably yes versus probably not + definitely not. 
Table 2. Reoperation categories.

\begin{tabular}{lcc}
\hline Reoperation Category & $\begin{array}{c}\text { D + ILS } \\
\mathbf{N}=\mathbf{7 7}) \\
\mathbf{n ~ ( \% )}\end{array}$ & $\begin{array}{c}\mathbf{D}+\text { Fusion } \\
\mathbf{( N = 3 9 )} \\
\mathbf{n ~ ( \% )}\end{array}$ \\
\hline Wound/surgery related & $3(3.9 \%)$ & $1(2.6 \%)$ \\
Under treatment & $2(2.6 \%)$ & $2(5.1 \%)$ \\
Device related issue & $2(2.6 \%)$ & $2(5.1 \%)$ \\
Device ineffective & $2(2.6 \%)$ & $1(2.6 \%)$ \\
$\quad$ A. Early ( $\leq 2$ years postop) & $1(1.3 \%)$ & $4(10.3 \%)$ \\
B. Late (>2 years postop) & 0 & 0 \\
Trauma & $10(13.0 \%)$ & $10(27.5 \%)$ \\
Total &
\end{tabular}

Abbreviations: D, decompression; ILS, interlaminar stabilization.

ence between groups at 60 months $(P>.7)$, it is notable that the ILS group achieved greater improvement in VAS leg pain scores than fusion and in less time. At 24 months, there was a statistically significant difference between groups, where the ILS group reported an average of 17.1 and the fusion group an average of $30.0(P=.035)$ (Figure $3 b$ ). Within both the ILS and the fusion groups, every follow-up mean VAS worse leg pain score was significantly better than baseline based on a within-group paired $t$ test (ILS $P<.001$, fusion $P$ $<.025)$.

\section{SF-12}

Within both groups, each follow-up mean SF-12 Physical Component Summary score was significantly better than baseline $(P<.001)$. In the ILS group, each follow-up mean SF-12 Mental Health Summary score was significantly better than baseline $(P<.001)$. In contrast, for the fusion group from month 36 through month 60 , scores were not significantly better than baseline $(P>.05)$. There was no significant difference between the groups' SF-12 Physical Component Summary and Mental
Health Summary scores at 60 months or at any other follow-up time point.

\section{$Z C Q$}

For the ZCQ, there was no significant difference between groups for Symptom Severity at 60 months $(P>.3)$ or at any other time point (Figure $4 a)$. The ZCQ Physical Functions scores, however, were significantly different between 2-level ILS patients and 2-level fusion patients at 6 weeks postoperation, with ILS patients having a mean score of 1.76 and fusion patients a mean score of $2.00(P=.039)$. At 24 months, the ILS group had a lower ZCQ mean Physical Function score than the fusion group again $(P=.056)$. At 5 years, there was no significant difference in ZCQ Physical Function scores between the ILS and fusion patient groups $(P>.7)$ (Figure $4 \mathrm{~b})$. For the ZCQ Satisfaction score, there was no significant difference between patient satisfaction at month 60; however, there were significant differences between 2-level ILS and 2-level fusion patients at week $6(P=.029)$ and at month $24(P=.009)$ (Figure $4 c)$. For both Symptom Severity and Physical Function, each follow-up mean ZCQ score was significantly better than baseline, based on a withingroup paired $t$ test $(P<.001)$.

\section{Narcotics Usage}

There was no significant difference between treatments in the number of patients using narcotics for pain management at any follow-up time point for any class of narcotics (month $60 P>.8$ ). There was also no significant difference between treatments in the number of patients using nonsteroidal anti-inflammatory drugs, acetylsalicylic acid, or
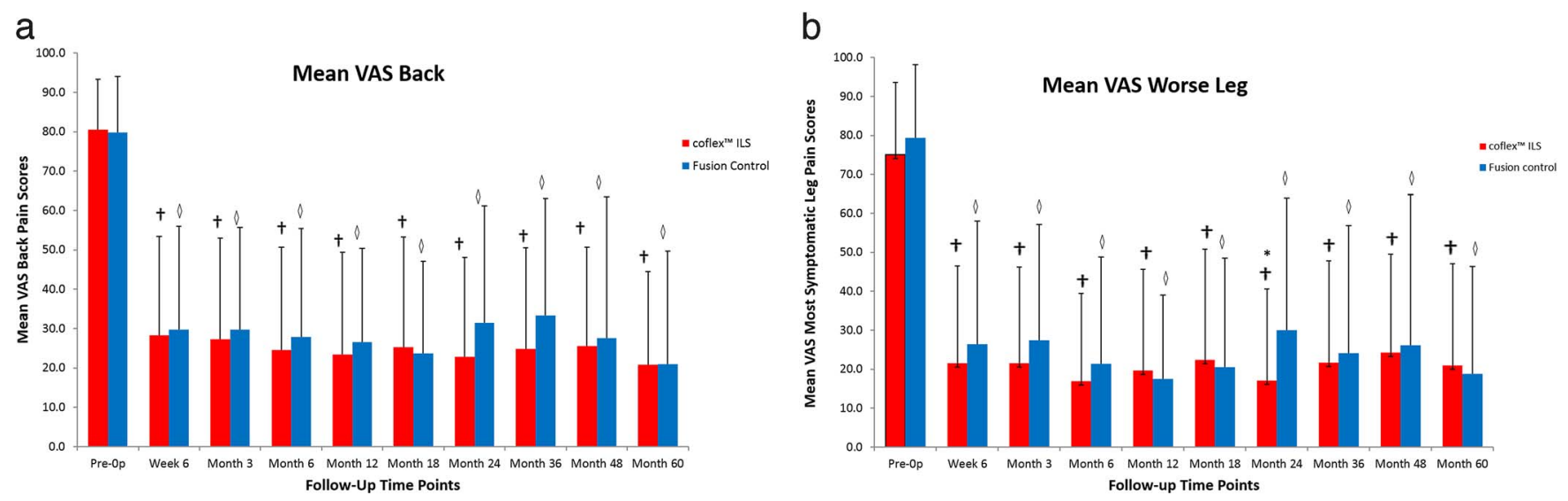

Figure 3. (a) Mean Visual Analog Scale (VAS) back pain scores. (b) Mean VAS worse leg pain scores. *Significant difference between interlaminar stabilization (ILS) and fusion groups: 2 sample pooled $t$ test $P$ value $=.035$. †Significant difference between ILS baseline and follow-up: within-group paired $t$ test. $\diamond$ Significant difference between fusion baseline and follow-up: within-group paired $t$ test. 

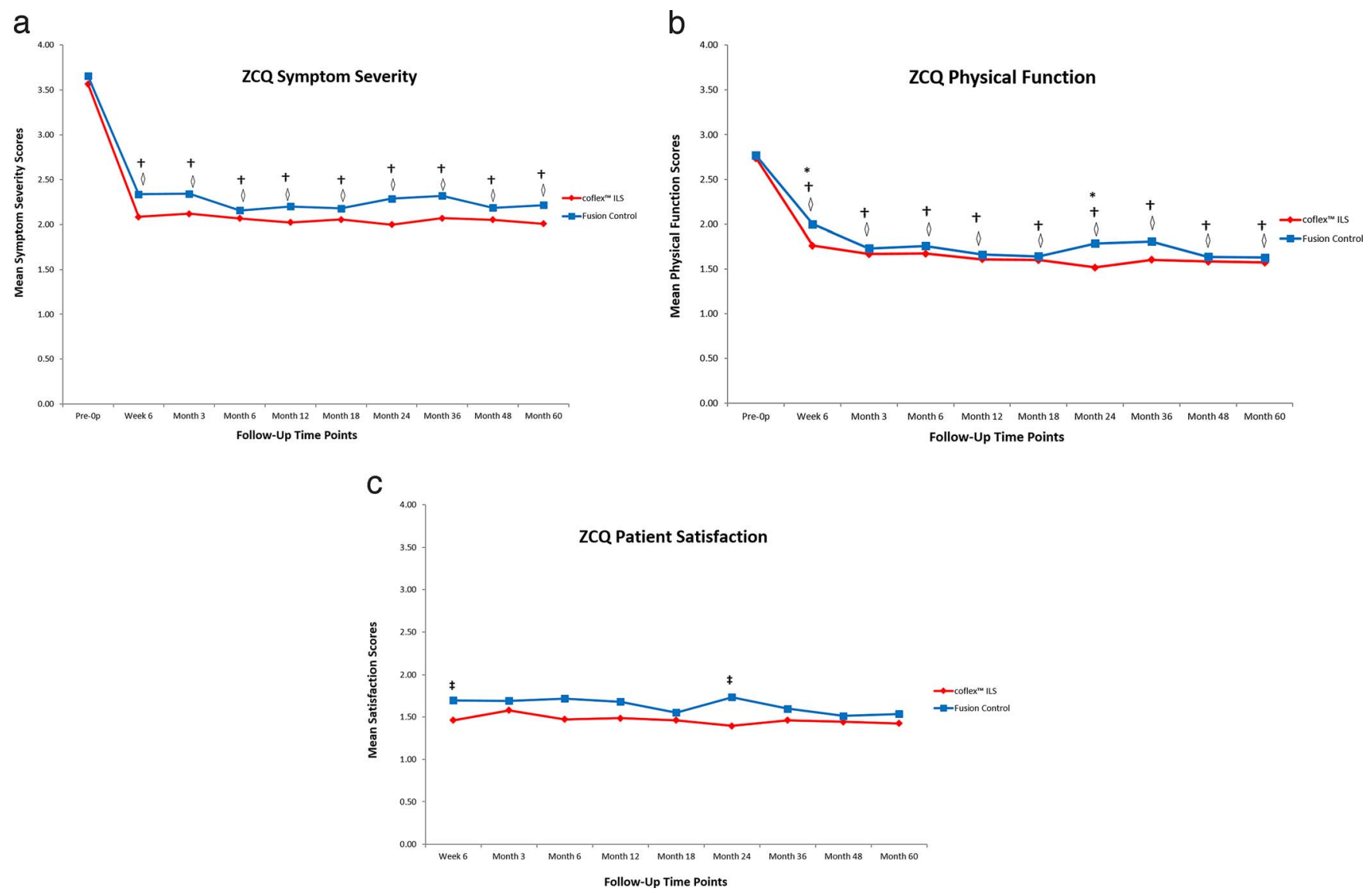

Figure 4. (a) Mean Zurich Claudication Questionnaire (ZCQ) symptom severity scores. (b) Mean ZCQ physical function scores. (c) Mean ZCQ patient satisfaction scores. *Significant difference between interlaminar stabilization (ILS) and fusion groups for Physical Function: 2 sample pooled $t$ test $P$ value at week $6=.039$, at month $24=.056$. $¥$ Significant difference between ILS and fusion groups for Patient Satisfaction: 2 sample pooled $t$ test $P$ value at week $6=.029$, at month $24=.009$. $\dagger$ †ignificant difference between ILS baseline and follow-up: within-group paired $t$ test. $\diamond$ Significant difference between fusion baseline and follow-up: within-group paired $t$ test.

acetaminophen for pain management at any followup time point (month $60 P>.4$ ).

\section{Radiological Assessment}

At every time point through month 60 , the average of the change in disc height from baseline, measured both anteriorly and posteriorly, between treatments was not different. The foraminal height (FH) was measured in the ILS group only. Preoperatively, the mean FH was $17.79 \pm 2.84$ $\mathrm{mm}$. There was no statistical difference at month 60 from baseline where the mean $\mathrm{FH}$ was $17.02 \pm 3.0$ $\mathrm{mm}$.

\section{Comparison of 1-Level ILS versus 2-Level ILS}

There was no statistically significant difference between 1-level and 2-level ILS patients with regard to improvement of ODI by $\geq 15$ points $(P=.172)$, the absence of reoperations or epidurals $(P=.911)$, the absence of persistent or new neurological deficits
$(P=.195)$, and the absence of major device-related complications $(P=.262)$. Of the ILS patients, $46.3 \%$ of 1-level and $55.1 \%$ of 2 -level patients met the criteria for CCS $(P=.246)$.

\section{DISCUSSION}

This is the first report specifically focused on the sustainability and longevity of interlaminar stabilization in multilevel surgery. This cohort analysis of a prospective randomized, multicenter study showed that ILS was not inferior to either posterolateral fusion or single-level ILS. The clinical and radiographic outcomes of 2-level ILS cases were at least as favorable as those of 2-level fusion cases, if not superior in some measures. With patients in both groups seeing statistically significant improvements across all outcome measures from baseline to 5 years postoperation, this analysis suggests that decompression with ILS is a viable surgical alternative to decompression with fusion in 2-level cases. 
Although the cohort study was not powered to find differences, the clinical composite score almost reached statistical significance and provides a strong trend toward superior outcomes with an ILS procedure. The most notable findings at the 5-year follow-up were with regard to the percentage of patients undergoing a secondary surgical intervention. The fusion group experienced secondary surgical interventions in $25.7 \%$ of patients, which was twice as high as the ILS group (13.0\%). When exploring the reasons for secondary surgery, the majority of reoperations in ILS patients were due to surgical complications not related to the device. As this was an IDE trial, it is expected to have some learning curve types of issues with new surgical procedures. In contrast, the most common reason for secondary surgery in fusion patients was "device-ineffective: post 24 month" or late-term revisions attributed mostly to either pseudarthrosis or hardware failure. This accounted for $10.3 \%$ of fusion patients in comparison to the $1.3 \%$ of ILS patients experiencing a late-term revision. Although the sample size for the fusion group was low, the trends seen in this analysis describe a significant advantage to ILS over fusion. Radiographic outcomes confirm that ILS performed as well as fusion in maintaining disc height and provided sustained preservation of foraminal height. Interlaminar stabilization, while preserving motion across the segment, was able to restore lordosis to the index level as well as fusion.

As spinal stenosis is a progressive, degenerative disease, there is a broad range of symptomatology, requiring a comprehensive approach. While relieving symptoms at the index level, focused treatment options that minimize complications and invasiveness and undo the mechanical burden on adjacent levels must be considered. The two most established surgical techniques are decompression alone or decompression with posterior fusion. Significant debate with varying levels of clinical evidence has been published to try to establish the boundaries of each approach. Recently, this journal published a policy statement with an extensive review of the literature to address this very issue. ${ }^{10}$ Briefly, decompression can be very effective in the absence of spondylolisthesis but is also associated with significant secondary interventions and dural tear incidence. ${ }^{1,11}$ However, in the presence of spondylolisthesis, decompression surgery alone repeatedly shows significantly lower intraoperative demand in terms of operative time and blood loss; however, the rates of postoperative instability resulting in high reoperation rates provide evidence against the technique. ${ }^{12-14}$ Including fusion after decompression to provide stability in spinal stenosis patients has become a widely accepted technique when spondylolisthesis is present and is more controversial when absent. Forsth et $\mathrm{al}^{12}$ prospectively studied spinal stenosis patients with and without degenerative spondylolisthesis and found no difference in clinical outcomes at 5-year follow-up between decompression alone compared to decompression and fusion. Ghogawala et $\mathrm{al}^{13}$ prospectively studied spinal stenosis patients with stable spondylolisthesis and found superior results with the addition of fusion to decompression surgery alone at a single level surgery. Although many previous investigations included bisegmental surgery $^{12,15-17}$ for the treatment of spinal stenosis, none of these previous studies reported the clinical outcomes for the multilevel experience separately or in comparison to single level. Therefore, we believe the current study offers the first analysis of the 2-level experience and demonstrates both clinical outcome success and favorably low reoperation rates in patients who received ILS surgery.

\section{REFERENCES}

1. Weinstein JN, Tosteson TD, Lurie JD, et al. Surgical versus nonsurgical therapy for lumbar spinal stenosis. $N$ Engl J Med. 2008;358(8):794-810.

2. Stromqvist BH, Berg S, Gerdhem P, et al. X-stop versus decompressive surgery for lumbar neurogenic intermittent claudication: randomized controlled trial with 2-year followup. Spine (Phila Pa 1976). 2013;38(17):1436-1442.

3. Patel VV, Whang PG, Haley TR, et al. Superion interspinous process spacer for intermittent neurogenic claudication secondary to moderate lumbar spinal stenosis: two-year results from a randomized controlled FDA-IDE pivotal trial. Spine (Phila Pa 1976). 2015;40(5):275-282.

4. Patel VV, Whang PG, Haley TR, et al. Two-year clinical outcomes of a multicenter randomized controlled trial comparing two interspinous spacers for treatment of moderate lumbar spinal stenosis. BMC Musculoskelet Disord. 2014;15:221.

5. Barbagallo GM, Olindo G, Corbino L, et al. Analysis of complications in patients treated with the $\mathrm{X}$-stop interspinous process decompression system: proposal for a novel anatomic scoring system for patient selection and review of the literature. Neurosurgery. 2009;65(1):111-119; discussion 119-120.

6. Davis RJ, Errico TJ, Bae H, et al. Decompression and coflex interlaminar stabilization compared with decompression and instrumented spinal fusion for spinal stenosis and lowgrade degenerative spondylolisthesis: two-year results from the prospective, randomized, multicenter, food and drug adminis- 
tration investigational device exemption trial. Spine (Phila Pa 1976). 2013;38(18):1529-1539.

7. Davis R, Auerbach JD, Bae H, et al. Can low-grade spondylolisthesis be effectively treated by either coflex interlaminar stabilization or laminectomy and posterior spinal fusion? two-year clinical and radiographic results from the randomized, prospective, multicenter US investigational device exemption trial: clinical article. $J$ Neurosurg Spine. 2013;19(2):174-184.

8. Musacchio MJ, Lauryssen C, Davis RJ, et al. Evaluation of decompression and interlaminar stabilization compared with decompression and fusion for the treatment of lumbar spinal stenosis: 5-year follow-up of a prospective, randomized, controlled trial. Int J Spine Surg. 2016;10:6.

9. Abjornson C, Yoon BJ, Callanan T, et al. Spinal stenosis in the absence of spondylolisthesis: can interlaminar stabilization at single and multi-levels provide sustainable relief? Int $J$ Spine Surg. 2018;12(1):64-69.

10. Guyer R, Musacchio M, Cammisa F, et al. ISASS recommendations/coverage criteria for decompression with interlaminar stabilization-coverage indications, limitations, and/or medical necessity. Int J Spine Surg. 2016;10.

11. Modhia U, Takemoto S, Braid-Forbes MJ, et al. Readmission rates after decompression surgery in patients with lumbar spinal stenosis among Medicare beneficiaries. Spine (Phila Pa 1976). 2013;38(7):591-596.

12. Forsth P, Olafsson G, Carlsson T, et al. A randomized, controlled trial of fusion surgery for lumbar spinal stenosis. $N$ Engl J Med. 2016;374(15):1413-1423.

13. Ghogawala Z, Dziura J, Butler WE, et al. Laminectomy plus fusion versus laminectomy alone for lumbar spondylolisthesis. N Engl J Med. 2016;374(15):1424-1434.

14. Son S, Kim WK, Lee SG, et al. A comparison of the clinical outcomes of decompression alone and fusion in elderly patients with two-level or more lumbar spinal stenosis. $J$ Korean Neurosurg Soc. 2013;53(1):19-25.

15. Kumar N, Shah SM, Ng YH, et al. Role of coflex as an adjunct to decompression for symptomatic lumbar spinal stenosis. Asian Spine J. 2014;8(2):161-169.

16. Roder C, Baumgartner B, Berlemann U, et al. Superior outcomes of decompression with an interlaminar dynamic device versus decompression alone in patients with lumbar spinal stenosis and back pain: a cross registry study. Eur Spine J. 2015;24(10):2228-2235.

17. Weinstein JN, Tosteson TD, Lurie JD, et al. Surgical versus nonoperative treatment for lumbar spinal stenosis fouryear results of the spine patient outcomes research trial. Spine (Phila Pa 1976). 2010;35(14):1329-1338.

Disclosures and COI: The fourth author is a member of the Corporate Medical Advisory Board, owns stock options and warrants, owns equity investment in non-publicly traded investment, and has a consulting agreement with a company involved in the manufacture of the device in this study. The fifth author receives compensation for consulting services from a company involved in the manufacture of the device in this study.

Corresponding Author: Christina Dowe, BS, Integrated Spine Research Program, Hospital for Special Surgery, New York, NY 10021. Phone: (917) 260-4285; Fax: (212) 606-1746; Email: dowec@hss.edu.

Published 31 August 2018

This manuscript is generously published free of charge by ISASS, the International Society for the Advancement of Spine Surgery. Copyright (C) 2018 ISASS. To see more or order reprints or permissions, see http://ijssurgery.com. 